\title{
Mesoscopic Charge Density Wave in a Magnetic Flux
}

\author{
Gilles Montambaux \\ Laboratoire de Physique des Solides, associé au CNRS \\ Université Paris-Sud \\ 91405 Orsay, France
}

(May 18, 2017)

\begin{abstract}
The stability of a Charge Density Wave (CDW) in a one-dimensional ring pierced by a AharonovBohm flux is studied in a mean-field picture. It is found that the stability depends on the parity of the number $N$ of electrons. When the size of the ring becomes as small as the coherence length $\xi$, the CDW gap increases for even $N$ and decreases for odd $N$. Then when $N$ is even, the CDW gap decreases with flux but it increases when $N$ is odd. The variation of the BCS ratio with size and flux is also calculated. We derive the harmonics expansion of the persistent current in a presence of a finite gap.
\end{abstract}

\section{INTRODUCTION}

It has been proposed recently that a Aharonov-Bohm (AB) flux $\phi$ should affect the stability of a Charge Pensity Wave (CDW) in a one-dimensional ring geometry 1 , 3 as it may have been seen in a recent experiment on CDH pierced by AB flux lines trapped in columnar defectst. One possible cause for the modulation of the CDW stability is the discreteness of the spectrum and the module tion of the position of the energy levels with the flux 2,3 . Indeed, it has been predicted that the CDW gap and the critical temperature oscillate with the flux $\phi$, with a period $\phi_{0}=h / e$ and that they are maxima at $\phi=0$ and minima at $\phi=\phi_{0} / 2$. When the perimeter $L$ of the ring becomes of the order of the correlation length $\xi$, i.e. when the CDW order parameter becomes of the order of the mean level spacing, the CDW can even bedestroyed when $\left|\phi-n \phi_{0}\right|$ is larger than a critical value 2.3 .

In this paper, we elaborate on these ideas and we show that the stability of the CDW depends crucially on the parity of the number $N$ of particles in the 1D ring (considering here spinless particles), an effect which has not been properly considered in previous works. When this number $N$ is odd, the CDW is destabilized by decreasing the length of the ring. But when $N$ is even, it we find that the CDW is stabilized. When $N$ is even, the effect of an $\mathrm{AB}$ flux is to destabilize the CDW, as found in refs. But when $N$ is odd, we find that the CDW can be stabilized by the flux, contrary to the conclusions of refs.2.3. This effect is reminiscent of the parity effect for the persistent current in one-dimensional rings 5 , 6 . In the following, we shall consider the case of spinless electrons.

In the next section, we establish the thermodynamic equations for the CDW in a finite 1D system, with emphasis on the parity effect. In section III, we calculate the flux variation of the order parameter in the form of a Fourier series. In section IV, the same is done for the critical temperature. Finally we calculate the persistent current in the last section.

\section{CDW IN A MAGNETIC FLUX}

Consider a one-dimensional ring of perimeter $L$. The periodic boundary conditions fix the wave vector of the eigenstates. In the presence of a flux, the possible wave vectors are:

$$
k=\frac{2 \pi}{L}(p+\varphi)
$$

where $\varphi$ is the dimensionless flux $\phi / \phi_{0}$ and $p \in \mathcal{Z}$. Let $N$ be the total number of electrons. The Fermi wave vector $k_{F}$ is:

$$
k_{F}=\frac{N \pi}{L}
$$

and the Fermi energy $\epsilon_{F}$ is:

$$
\epsilon_{F}=\frac{\hbar^{2}}{2 m}\left(\frac{N \pi}{L}\right)^{2} .
$$

It is important to stress that with this choice of $k_{F}$ and $\epsilon_{F}$, the number of electrons is independent of the flux, Fig.(1).

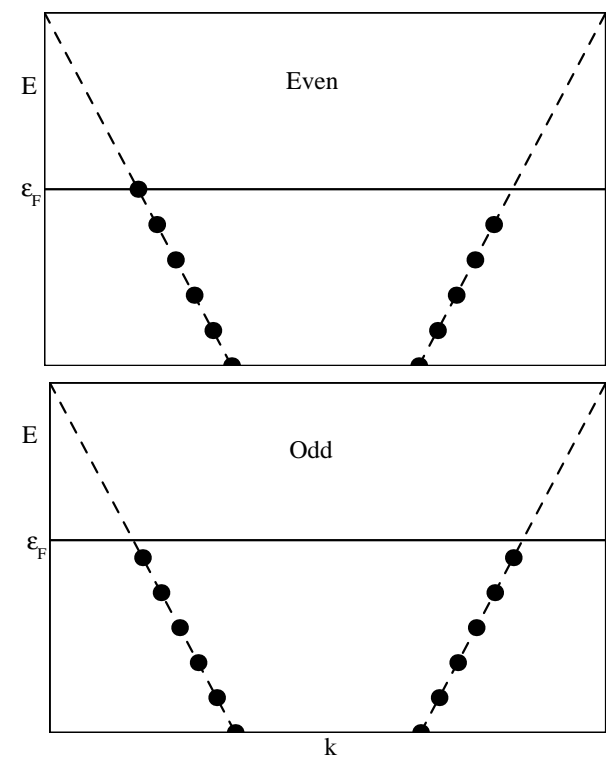


FIG. 1. Dispersion relation in the metallic phase, a) when the number $N$ of particles is even (do not forget the state $k=0$ ), b) when $N$ is odd. The horizontal line gives the position of the Fermi level.

Although the chemical potential is in principle fixed by a reservoir in ref.E, we shall see later that their results correspond actually to a fixed even number of particles.

The theory of the CDW ordering is well known. In the presence of a periodic potential with wave vector $Q$, the Hamiltonian is:

$$
\mathcal{H}=\mathcal{H}_{0}+2 \Delta \cos (Q r+\theta)
$$

where the eigenenergies $\epsilon_{k}$ of $\mathcal{H}_{0}$ are known. Because of the periodic potential, the states $k$ are in principle coupled to states $k \pm n Q$ where $n=1,2,3, \ldots$. We consider the case of a weak potential. Neglecting commensurability effects, one has to diagonalize a $2 \times 2$ matrix: the states with wave vector $k \simeq k_{F}$ are coupled to states with wave vector $k-Q \simeq-k_{F}$ :

$$
\left(\begin{array}{lc}
\epsilon(k) & \Delta e^{i \theta} \\
\Delta e^{-i \theta} & \epsilon(k-Q)
\end{array}\right)
$$

The eigenvalues are given by $E=\frac{\epsilon_{k}+\epsilon_{k-Q}}{2} \pm$ $\sqrt{\left(\frac{\epsilon_{k}-\epsilon_{k-Q}}{2}\right)^{2}+\Delta^{2}}$. It is then convenient to linearize the dispersion near the Fermi level so that, by taking the origin of the energies at the Fermi level, one has:

$$
\epsilon_{k}=\hbar v_{F}\left(|k|-k_{F}\right)=2 \delta\left(|p+\varphi|-\frac{N}{2}\right)
$$

where the mean level spacing $\delta$ near the Fermi level is given by $\pi \hbar v_{F} / L$. Writing the nesting vector $Q$ in the form $Q=2 \pi q / L$, where $q$ is an integer, the eigenvalues in the CDW phase are:

$$
E_{p}=(q-N) \delta \pm \sqrt{4 \delta^{2}\left(p-\frac{q}{2}+\varphi\right)^{2}+\Delta^{2}}
$$

The nesting condition implies $q=N$ so that the nesting vector is

$$
Q=2 k_{F}=\frac{2 N \pi}{L}
$$

and the energy levels in the CDW phase become:

$$
\begin{aligned}
E_{p} & = \pm \sqrt{4 \delta^{2}\left(p-\frac{N}{2}+\varphi\right)^{2}+\Delta^{2}} \\
& = \pm 2 \delta \sqrt{\left(p-\frac{N}{2}+\varphi\right)^{2}+\Lambda^{2}}
\end{aligned}
$$

where $\Lambda=\Delta /(2 \delta)$. $\quad \Lambda$ measures the gap amplitude in units of the mean level spacing and can be written as the ratio of the length over the CDW coherence length $\xi=\hbar v_{F} / \pi \Delta$. One has $\Lambda=L /\left(2 \pi^{2} \xi\right)$. The effects discussed in this paper appear for small rings when the mean level spacing becomes as large as the the CDW gap, see Fig.(2).

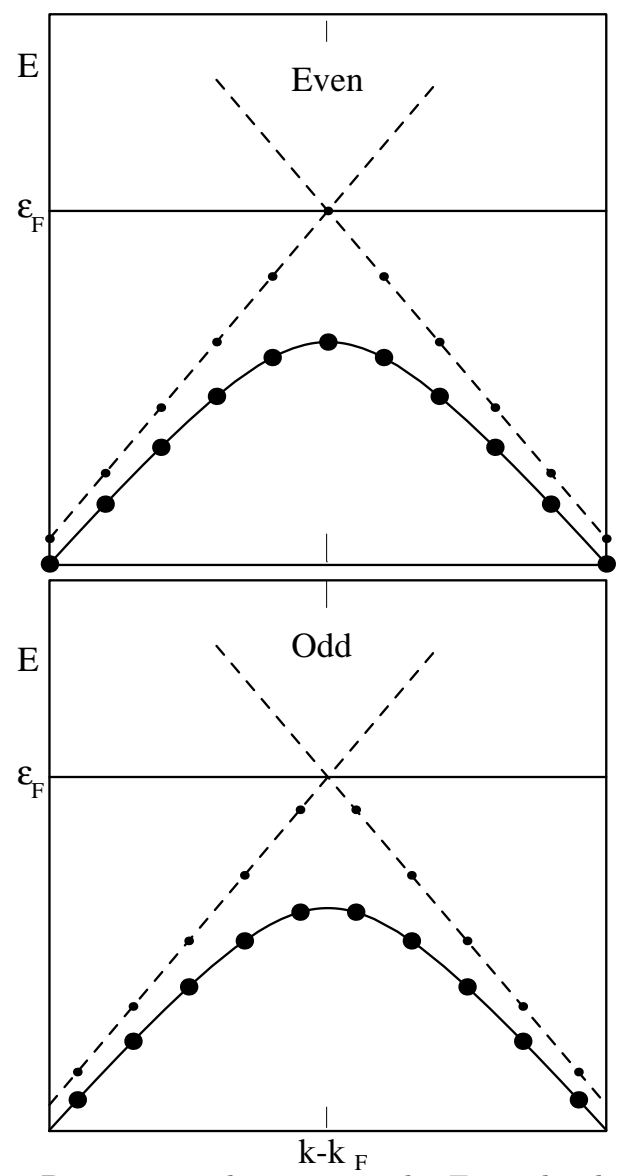

FIG. 2. Dispersion relation near the Fermi level, a) when the number $N$ of particles is even, b) when $N$ is odd. The horizontal line gives the position of the Fermi level. The small dots denote the states in the metallic phase and the large dots represent the states in the CDW phase

From minimization of the total free energy

$$
F=\frac{\Delta^{2}}{\lambda} L-k_{B} T \log \mathcal{Z}
$$

where $\lambda$ is the interaction parameter and $\mathcal{Z}$ is the grand canonical partition function, the well-known selfconsistency condition is obtained:

$$
1=\sum_{p} \frac{g}{2 E_{p}} \tanh \left(\frac{\beta}{2} E_{p}\right)
$$

where $g=\lambda / 2 \pi \hbar v_{F}$. When $N$ is even, the introduction of a new variable $n=p-N / 2$ reduces this equation to:

$$
1=g \sum_{n} \frac{\tanh \left(\frac{\beta^{\prime}}{2} \sqrt{(n+\varphi)^{2}+\Lambda^{2}}\right)}{2 \sqrt{(n+\varphi)^{2}+\Lambda^{2}}}
$$

where $\beta^{\prime}=2 \beta \delta$. This is the result found in ref. 2 . However, when the number $N$ is odd, $n=p-(N-1) / 2$ and the self-consistency equation becomes: 


$$
1=g \sum_{n} \frac{\tanh \left(\frac{\beta^{\prime}}{2} \sqrt{\left(n-\frac{1}{2}+\varphi\right)^{2}+\Lambda^{2}}\right)}{2 \sqrt{\left(n-\frac{1}{2}+\varphi\right)^{2}+\Lambda^{2}}}
$$

Thus the stability of the $C D W$ depends on the parity of the number of electrons. The flux dependence for the even and odd parities are deduced from each other by a trapslation of $\phi_{0} / 2$. We stress the fact that the results of refl 6 correspond to a fixed even number of particles, once the chemical potential is fixed to the value of eq.(1). If the chemical potential was fixed to any other value, the number of particles would vary with the field leading to discontinuities of the persistent current $\bar{b}$ and of the CDW stability.

\section{GROUND STATE}

We first study the evolution of the CDW order parameter $\Delta_{\varphi}$ at zero temperature, as a function of the size and of the AB flux. When $N$ is even, it is given by

$$
1=\frac{g}{2} \sum_{n} \frac{1}{\sqrt{(n+\varphi)^{2}+\Lambda_{\varphi}^{2}}}
$$

where the dimensionless parameter $\Lambda_{\varphi}=\Delta_{\varphi} / 2 \delta$ has been introduced. This sum diverges at large $n$ and is usually cut-off at an energy scale $E^{*}$ of the order of the bandwidth. This corresponds to an integer $n^{*}=E^{*} / 2 \delta$. In ref.2, the flux dependence of the order parameter is given by:

$$
\sum_{n}\left[\frac{1}{\sqrt{(n+\varphi)^{2}+\Lambda_{\varphi}^{2}}}-\frac{1}{\sqrt{n^{2}+\Lambda_{0}^{2}}}\right]=0
$$

which corresponds to the case where $N$ is even. However, when $N$ is odd, $n$ should be replaced by $n-1 / 2$ as seen in eq.(四).

We found it convenient to write the Fourier decomposition of the flux dependence of the order parameter. To transform eq.(5), we use the Poisson summation formula

$$
\sum_{n} f(n+\varphi)=g_{0}+2 \sum_{m>0} g_{m} \cos (2 \pi m \varphi)
$$

where

$$
g_{m}=2 \int_{0}^{\infty} f(y) \cos (2 \pi m y) d y
$$

The constant term $g_{0}$ is divergent and must be cut-off with $n^{*}$. The Fourier components are convergent. As a result one gets:

$$
\frac{1}{g}=\ln \frac{2 E^{*}}{\Delta_{\varphi}}+2 \sum_{m>0}(-1)^{N m} K_{0}\left(2 \pi m \Lambda_{\varphi}\right) \cos 2 \pi m \varphi
$$

The factor $(-1)^{N m}$ in the harmonics expansion takes into account the parity effect. $K_{0}$ is a modified Bessel function of the second kinds.

It is instructive to consider first the ring in the absence of external flux. For the infinite bulk system, the gap $\Delta_{b}$ is given by:

$$
\frac{1}{g}=\ln \frac{2 E^{*}}{\Delta_{b}}
$$

When the size becomes finite, the gap in zero flux $\Delta_{0}$ is given by:

$$
\ln \frac{\Delta_{0}}{\Delta_{b}}=2 \sum_{m>0}(-1)^{N m} K_{0}\left(2 \pi m \frac{\Delta_{0}}{\Delta_{b}} \Lambda_{b}\right)
$$

where the parameter $\Lambda_{b}=\Delta_{b} / 2 \delta$ measures the bulk gap in units of mean level spacing and is proportional to the size.

The variation of the gap with $\Lambda_{b}=L / 2 \pi^{2} \xi_{b}$ is shown on Fig.(3). $\xi_{b}$ is the coherence length of the infinite system. When the number of particles is even, one sees that the gap increases with defreasing size. This seems to be in contradiction with refl who found a decrease of the critical temperature $T_{c}$ when the size decreases. We will comment on this point in the next section. When the number of particles is odd, the gap decreases with decreasing size. When the length becomes too small, the order parameter can even vanish. This happens for a critical value of $\Lambda_{b}=1 / 2 \gamma=.280 . \gamma$ is the Euler constant $\gamma=1.781$.

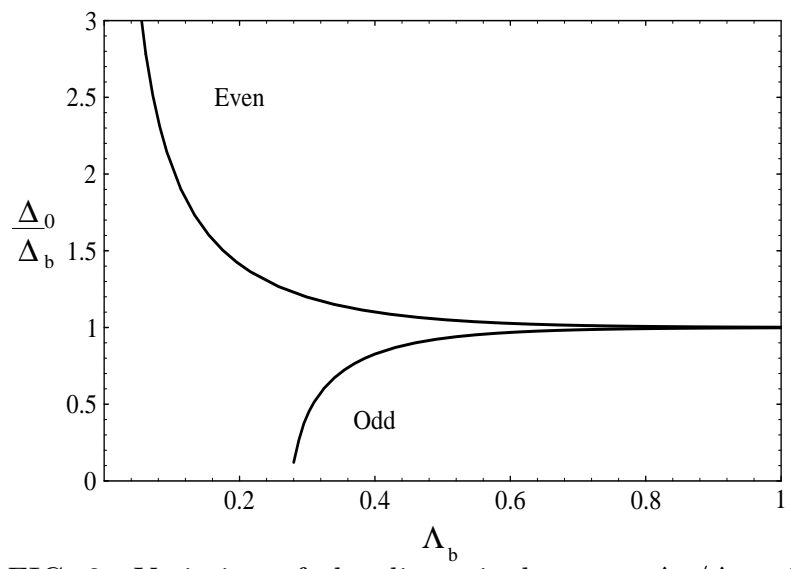

FIG. 3. Variation of the dimensionless gap $\Delta_{0} / \Delta_{b}$ with $\Lambda_{b}=\Delta_{b} / 2 \delta . \quad \Delta_{b}$ is the gap of the infinite system. $\Lambda_{b}$ is proportional to the size $L$.

We now turn to the effect of the AB flux. In a finite flux, the gap equation (9) transforms into:

$$
\ln \frac{\Delta_{\varphi}}{\Delta_{b}}=2 \sum_{m>0}(-1)^{N m} K_{0}\left(2 \pi m \frac{\Delta_{\varphi}}{\Delta_{b}} \Lambda_{b}\right) \cos 2 \pi m \varphi
$$

Fig.(14) shows the gap in the case of even $N$, for different fluxes. It is seen the effect of the flux is to reduce the gap, 
this effect being larger for small sizes. Actually the case $\varphi=1 / 2$ with an even number of particles is equivalent to the case $\varphi=0$ with an odd number of particles. This is obvious in Figs.(3) and (4) and in the structure of the gap equation (7).

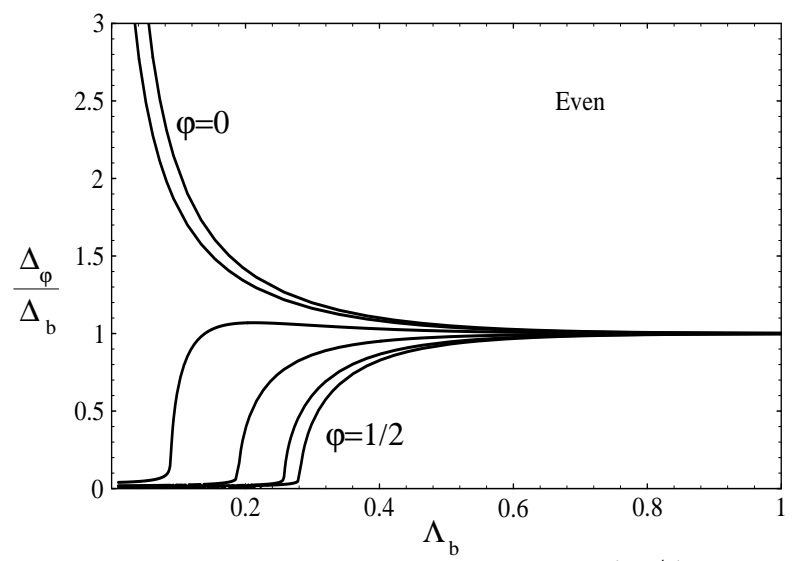

FIG. 4. Variation of the dimensionless gap $\Delta_{\varphi} / \Delta_{b}$ with $\Lambda_{b}$, for different fluxes $\varphi=0,0.1,0.2,0.3,0.4,0.5$.

Fig.(5) shows the flux dependence of $\Delta_{\varphi} / \Delta_{b}$ with the flux for different parameters $\Delta_{b} / 2 \delta \propto L / \xi_{b}$. For an even number of particles, the order parameter is enhanced at zero flux and is reduced for large flux. When $\Delta_{b} / 2 \delta$ becomes too small, the order parameter can even vanish near $\varphi=1 / 2$. This happens for a critical value of $\Lambda_{b}=1 / 2 \gamma=.280$. For smaller rings, i.e. when $\Lambda_{b}$ is smaller, the CDW disappears at a critical flux given by:

$$
\psi\left(\varphi_{c}\right)+\psi\left(1-\varphi_{c}\right)=2 \ln \Lambda_{b} / 2
$$

where $\psi$ is the digamma function.

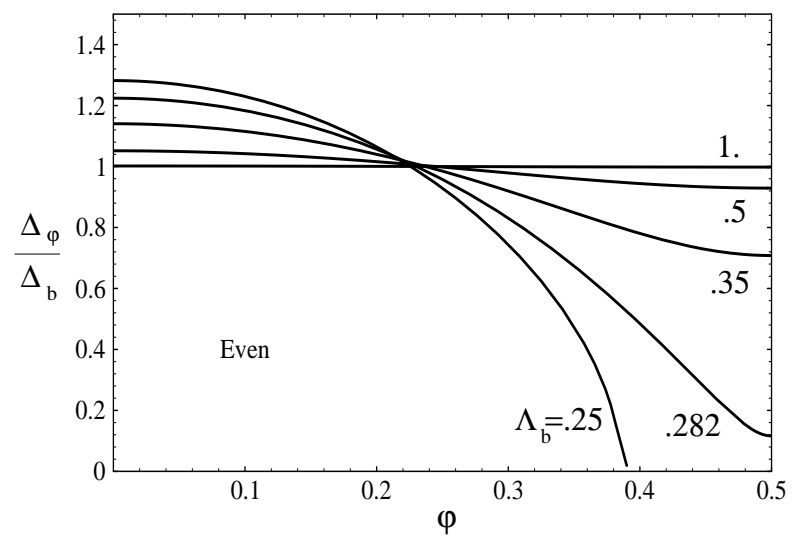

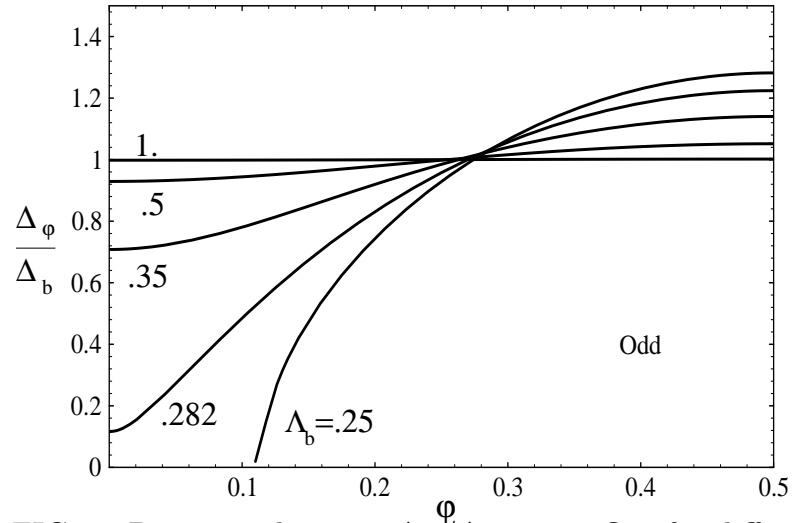

FIG. 5. Dimensionless gap $\Delta_{\varphi} / \Delta_{b}$ versus flux for different system sizes a) with even $N$, b) with odd $N$.

For the case of even N, the figure (5.a) is consistent with the figure 2 of ref.2 where $\Delta_{\varphi} / \Delta_{0}$ was plotted instead of $\Delta_{\varphi} / \Delta_{b}$ here. That figure could not show the interesting result that in low flux the gap increases when the size decreases.

For odd $N$, the order parameter increases in a finite magnetic flux and it is maximum at $\varphi=1 / 2$. This contradicts the arguments of ref.2 who argues that the suppression of the CDW order is due to pair-breaking induced by the field. There is indeed no pair-breaking effect since the field does not couple to the phase of the electronhole pair. The field effect here is simply to change the position of the energy levels and thus to either reduce or enhance the stability of the CDW. This can be simply understood from the schematic Figs.(2).

For a ring of large size, the modulation of the gap given by eq. 10 becomes weak and harmonic. It is given by:

$$
\Delta_{\varphi}=\Delta_{b}\left(1 \pm \frac{1}{\sqrt{\Lambda_{0}}} e^{-2 \pi \Lambda_{0}} \cos 2 \pi \varphi\right)
$$

which explicitely displays the exponential decrease of the modulation with the size of the ring.

\section{TRANSITION TEMPERATURE}

The dependence of the critical temperature with the size and the flux reflects those of the gap.

At the transition, $\Delta=\Lambda=0$. As in ref.2, the selfconsistency equation can be written as, for even $N$ :

$$
\frac{1}{g}=\frac{1}{2} \sum_{n} \frac{\tanh \left[\frac{\beta_{\varphi}^{\prime}}{2}(n+\varphi)\right]}{n+\varphi}
$$

Doing the same Poisson summation as above, the self consistency equation for the critical temperature $T_{\varphi}$ is found to be, taking into account the parity:

$$
\frac{1}{g}=\ln 1.14 \frac{E^{*}}{T_{\varphi}}+\sum_{m>0}(-1)^{N m} F\left(m / \beta_{\varphi}^{\prime}\right) \cos (2 \pi m \varphi)
$$


where the function $F$ is:

$$
F(x)=\ln \left(\frac{\cosh \left(2 \pi^{2} x\right)+1}{\cosh \left(2 \pi^{2} x\right)-1}\right)
$$
by:

The critical temperature of the infinite system is given

$$
\frac{1}{g}=\ln 1.14 \frac{E^{*}}{T_{b}}
$$

so that by difference between eqs.(11) and (12), one has the flux dependence of $T_{\varphi} / T_{b}$ for different parameters $\Lambda_{b}=\Delta_{b} / 2 \delta$ :

$$
\ln \frac{T_{\varphi}}{T_{b}}=\sum_{m>0}(-1)^{N m} F\left(\frac{m}{1.76} \Lambda_{b} \frac{T_{\varphi}}{T_{b}}\right) \cos 2 \pi m \varphi
$$

The result is shown on Fig.(6). For zero flux, the variation is very similar to that of the gap (Fig.3). When the size decreases, the critical temperature increases, in apparent contradiction with the figure (4) of ref.2 who found a decrease of the critical temperature. However their temperature is normalized to the gap, $\Delta_{0}$ which is itself size dependent, and not to $\Delta_{b}$. What is actually found in refl 2 is an increase of the BCS ratio $\Delta_{\varphi} / T_{\varphi}$. This is in agreement with our calculation of this ratio shown in Fig.(7) and exhibits the $12 \%$ increase found in ref.l.

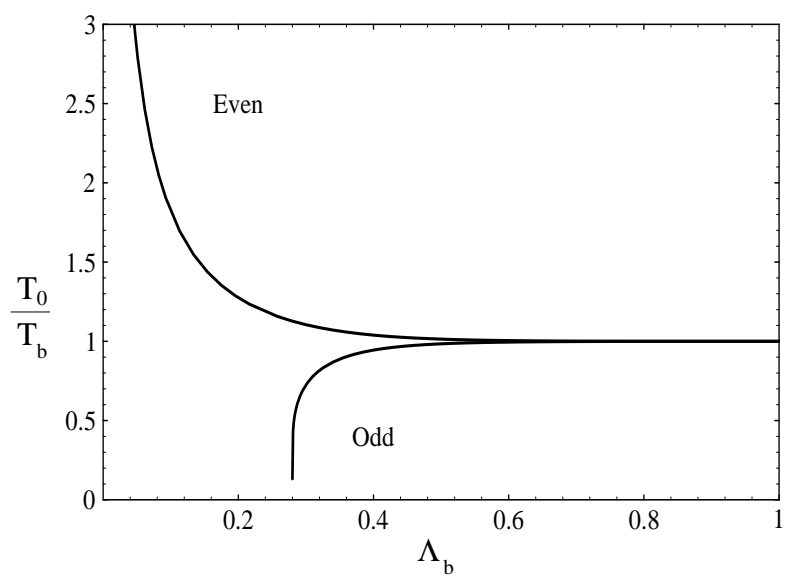

FIG. 6. Variation of the critical temperature with the size, in zero flux.

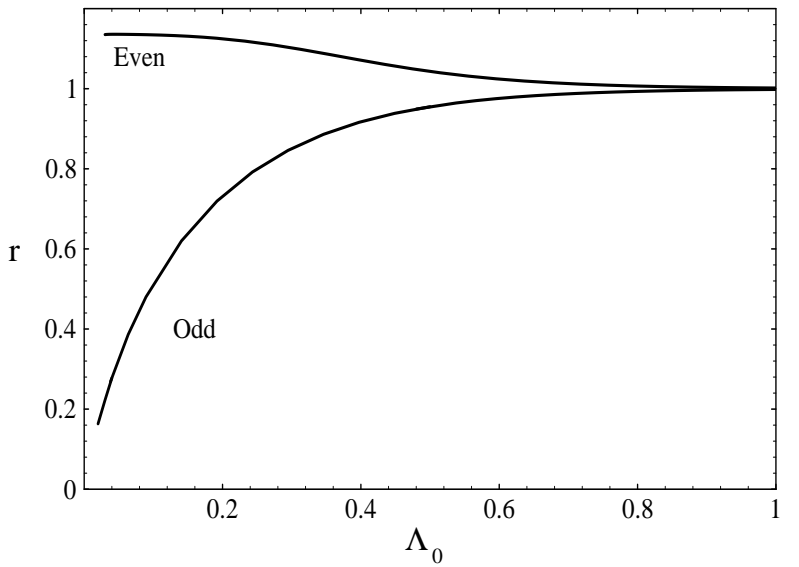

FIG. 7. Variation of the normalized BCS ratio $r=\left(\Delta_{0} / T_{0}\right) /\left(\Delta_{b} / T_{b}\right)$ with the size, in zero flux

Finally, we plot in Fig. (8) the variation of the critical temperature with the flux, for different sizes.

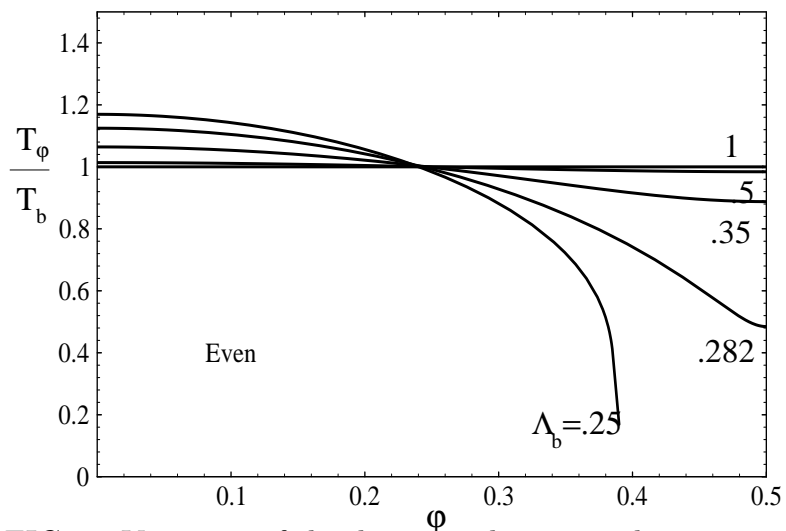

FIG. 8. Variation of the dimensionless critical temperature $T_{\varphi} / T_{b}$ with the flux, for different system sizes, when $N$ is even. The case where $N$ is odd deduces by the same symmetry as in Fig. 5

In the limit of a large system, the oscillations of the critical temperature become exponentially small as:

$$
T_{\varphi}=T_{b}\left(1 \pm 2 e^{-1.14 L / \xi} \cos 2 \pi \varphi\right)
$$

\section{PERSISTENT CURRENT}

The persistent current in the CDW phase is given by:

$$
I(\varphi)=-\sum_{n} 2 \frac{I_{0}}{N}(n+\varphi)+\sum_{n} I_{0} \frac{n+\varphi}{\sqrt{(n+\varphi)^{2}+\Lambda^{2}}}
$$

where $n \in[-N / 2, N / 2-1]$ if $N$ is even and $n \in[-(N-$ $1) / 2,(N-1) / 2]$ if $N$ is odd.

$$
I_{0}=\frac{2 \delta}{\phi_{0}}=\frac{e v_{F}}{L}
$$


is the maximal current in one dimension. Eq.(14) for the persistent current is exact for a quadratic dispersion relation 9 . The first term is the persistent current $I_{N}(\varphi)$ in the normal state: $I_{N}(\varphi)=-2 I_{0} \varphi$ when $N$ is odd and $I_{N}(\varphi)=-I_{0}(2|\varphi|-1)$ when $N$ is even 10

After summation by parts and Poisson summation, the flux dependence of the total current can be conveniently cast in the Fourier expansion which is parity dependent:

$$
I(\varphi)=4 I_{0} \Lambda_{\varphi} \sum_{m>0}(-1)^{N m} K_{1}\left(2 \pi m \Lambda_{\varphi}\right) \sin 2 \pi m \varphi
$$

$K_{1}$ is a modified Bessel function of the second kindl The persistent current depends on the gap which is itself flux dependent. For pedagogical purpose, we first show the flux variation of the current assuming a constant gap, Fig.(9).

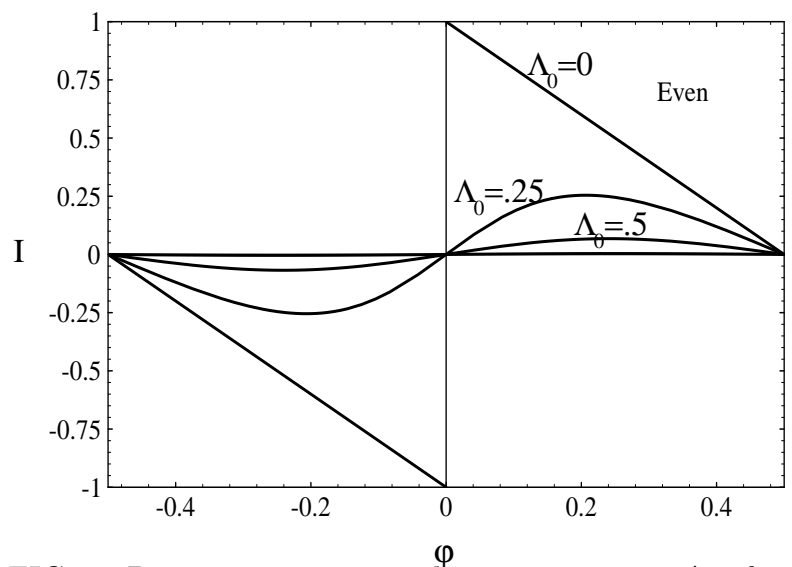

FIG. 9. Persistent current with a constant gap $\Delta_{0}$, for an even number of particles.

When the gap goes to zero, $K_{1}(x) \overrightarrow{1 / x}$ and one recovers the current of the normal statel

$$
I(\varphi)=\frac{2}{\pi} I_{0} \sum_{m>0} \frac{(-1)^{N m}}{m} \sin 2 \pi m \varphi
$$

When the gap becomes larger than the interlevel spacing, the current is reduced exponentially as:

$$
I(\varphi)=(-1)^{N} I_{0} e^{-1.14 L / \xi} \sin 2 \pi \varphi
$$

The variation of the gap itself with the flux must be taken into account. Fig.(10a) shows the variation of the current with the flux for $\Lambda_{0}=.35$. When the flux increases, the gap decreases so that the current becomes larger (full line) than if the gap were constant (dashed line). When $\Lambda_{0}=.25$, the CDW gap vanishes at a critical flux and the current recovers continuously its value in the metallic phase. This results constradicts those of ref. 3 who found a discontinuity in the current. When $N$ is odd, the current is trivially shifted by half a period $\phi_{0} / 2$, at variance with the conclusion of ref 3 .
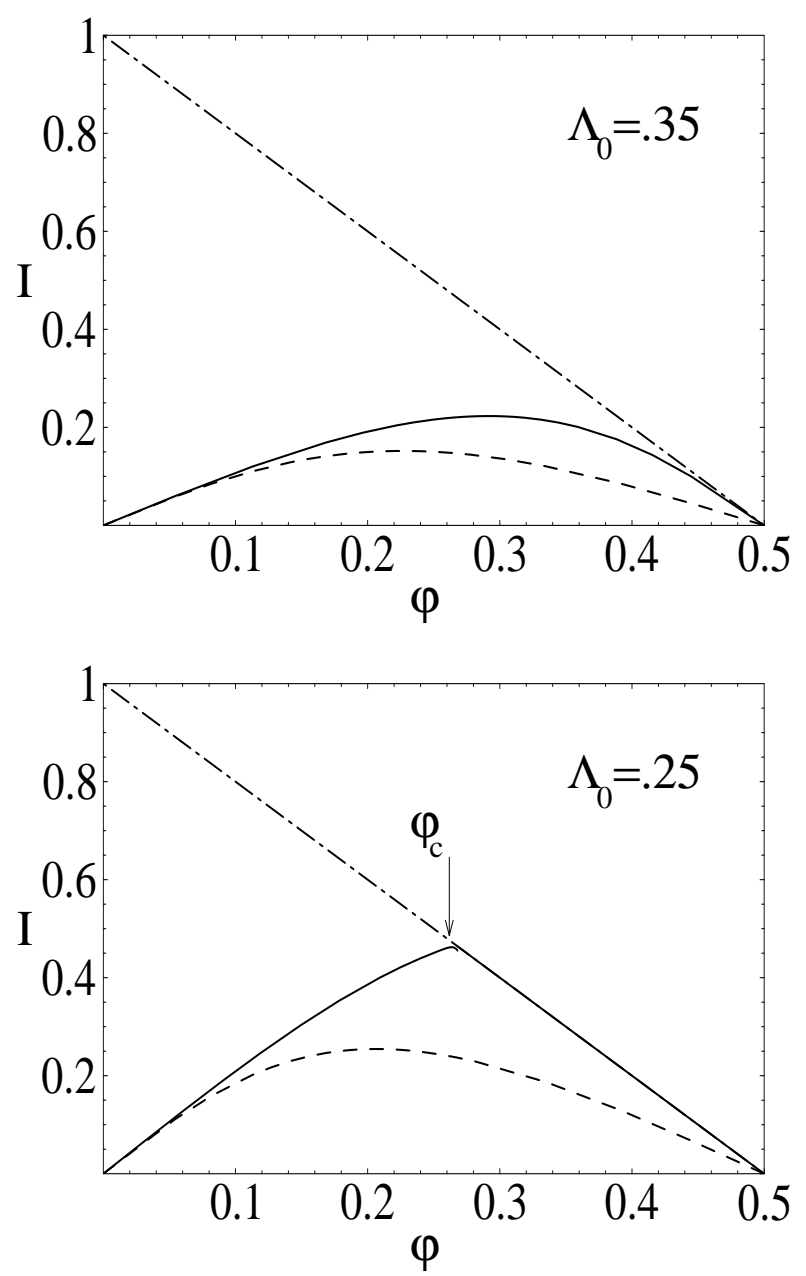

FIG. 10. Persistent current in the CDW phase (full line), for two values of the size $L$, in the case of even $N$. The dashed line shows the current if the gap were constant and the dotted-dashed line shows the current in the metallic phase.

\section{CONCLUSIONS}

We have derived the mean-field thermodynamics of a CDW in a small 1D clean system in the presence of a magnetic flux. The stability of the CDW depends on the parity of the number $N$ of particles. When the size decreases and becomes of the order of the coherence length $\xi=\hbar v_{F} / \pi \Delta$, the CDW order parameter increases if $N$ is even, it decreases if $N$ is odd. The CDW is stabilized by the magnetic flux when $N$ is odd and it is destabilized when $N$ is even. These results correct those of refs 2 . 3 who found that the flux always tends to suppress the Peierls instability.

These are the results for a one dimensional ring. They can be in principle generalized to the case of a many channel ring. Refl 3 suggests that the current is simply multiplied by the number of chains. This is not true, as it is already known for the metallic phase that the current results from a subtle addition of the contributions 
of the different channels 11. Such rings with few number of channels can be synthetized using thin-film growth of blue bronze oxydes 2 , 13 . The case of a 1D ring with short range interaction and impurities a disordered Luttinger liquid, has been studied recently 4 . It would be interesting to see how the discretness of the spectrum affects the obtained results.

Note added in proof: After this paper was accepted, I have been informed by F. Von Oppen of the existence of a related work with similar conclusions 15 . Here I have found the analytical expressions of the harmonics expansion of the critical temperature, gap and persistent current They agree with previous numerical calculations. Ref. 15 addresses the fluctuation effect.
${ }^{1}$ E.N. Bogachek, I.V. Krive, I.O. Kulik and A.S. Rozhavsky, Phys. Rev. B 42, 7614 (1990)

${ }^{2}$ M.I. Visscher, B. Rejaei and G.E.W. Bauer, Europhys. Lett. 36, 613 (1996).

${ }^{3}$ J. Yi, M.Y. Choi, K.Park and E.-H. Lee, Phys. Rev. Lett. 78, 3523 (1997).

${ }^{4}$ Yu. Latyshev, O. Laborde, P. Monceau and S. Klaumünzer, Phys. Rev. Lett. 78, 919 (1997)

${ }^{5}$ N. Trivedi and D.A. Browne, Phys. Rev. B 38, 9581 (1988)

${ }^{6}$ H.F. Cheung, Y. Gefen, E. Riedel and W. Shih, Phys. Rev. B 37, 6050 (1988)

${ }^{7}$ A.J. Leggett, in Granular nanoelectronics, Vol. 251, NATO ASI D.K. Ferry, J.R. Barker and C. Jacobini eds. (Plenum New-York 1991), p.297

8 The modified Bessel functions of the second kind, $K_{\nu}(x)$, are given by: $K_{\nu}(x)=\int_{0}^{\infty} e^{-x \cosh t} \cosh (\nu t) d t$. Handbook of Math. Funct., M. Abramovitz and I. Stegun (Dover).

${ }^{9}$ A linear dispersion relation for the normal phase would give a zero current in the normal phase.

${ }^{10}$ ref 2 confuses the expression of the current for even and odd parities.

${ }^{11}$ H.F. Cheung, Y. Gefen and E. Riedel, IBM J. Res. Develop. 32, 359 (1988)

12 H.S.J. van der Zant, O.C. Mantel, C. Dekker, C. Traeholt and H.W. Zandbergen, Phys. Rev B 55, 4017 (1996)

${ }^{13}$ O.C. Mantel, H.S.J. van der Zant, A.J. Steinfort, C. Dekker, J.E. Mooij and C. Traeholt, App. Phys. Lett. 68, 3823 (1996)

14 T. Giamarchi and B.S. Shastry, Phys. Rev. B 51, 10915 (1995)

15 B. Nathanson, O. Entin-Wohlman and B. Mühlschlegel, Phys. Rev. B 45, 3499 (1992) 